\title{
Isolating the components of very-short-term visual memory
}

\author{
JOHN PALMER \\ University of Washington, Seattle, Washington
}

\begin{abstract}
Very-short-term visual memory has often been measured with partial report, which uses attentional cues to measure performance as a function of cue delay. The resulting cue-delay effect is often accounted for by a limited ability to transfer information between a high-capacity and a low-capacity store. According to this transfer hypothesis, the performance measured in a typical partial report experiment results from a mixture of two components: information about the tested stimuli is transferred both before and after the cue. In the present experiment, the two components were isolated as follows. Observers attended and memorized a particular object in a multiple-object display. This display was followed by an auditory cue that indicated which of the stimuli would be tested. The critical comparison was between a stay condition, in which the auditory cue indicated the already attended object, and a switch condition, in which the auditory cue indicated a different object. The stay versus switch manipulation had a large effect. The stay condition had little cue-delay effect, whereas the switch condition yielded the usual cue-delay effect. This difference demonstrates the existence of multiple components in the processing and/or memory underlying partial report experiments.
\end{abstract}

In experiments on very-short-term visual memory, indirect methods are used to measure memory over fractions of a second following a visual stimulus. In the most common such paradigm, one uses partial sampling to measure memory for one part of the stimulus. The critical manipulation is to present a cue that indicates what part of the display is to be tested. For example, in the original partial report task (Sperling, 1960), a display of several rows of letters was followed by an auditory cue indicating which row of letters was to be reported. Increasing the delay between the display offset and the auditory cue reduced performance. This cue-delay effect was interpreted as providing an indirect measure of memory at the time of the cue. Partial sampling has also been adapted to a discrimination paradigm (Palmer, 1988; see also Kinchla, 1973). A stimulus of four lines was followed by an auditory cue, which was followed by a comparison line. The observer compared the length of the comparison line to the line in the corresponding position of the first stimulus. The cue provided an early indication about which line would be tested. This second partial sampling task also showed an effect of cue delay.

According to one interpretation of these partial sampling tasks, transfer occurs between two proposed memory stores (Rumelhart, 1970; Sperling, 1960)-a shortlived high-capacity store, and a more durable low-capacity

I thank Cynthia Ames, Corlene Ankrum, Beth Kerr, Geoff Loftus, Ray Slettevold, Penny Yee, and Zelda Zabinsky. This work was supported in part by a grant from the Graduate School of the University of Washington (PHS RR-07096). Correspondence should be sent to John Palmer, Department of Psychology, NI-25, University of Washington, Seattle, WA 98195. store. Information from a display is originally present in the high-capacity store; it must be transferred to the lowcapacity store if a response is to be mediated. The cue in the partial sampling task indicates what information must be transferred between the stores. If one assumes that no transfer occurs before the cue, and if the cue is processed instantaneously, the cue delay provides an indication of the information available in the high-capacity store at the time of the cue. This is the simplest form of what I will call the transfer hypothesis.

An extension of the simplest transfer hypothesis dates from the original article on partial report (Sperling, 1960). Suppose some information was transferred before the cue. Such a transfer would be particularly useful if the cue was considerably delayed, and any information would be better than none. If such a strategy was followed, then any response would be based on a mixture of two components: one in which the information was transferred before the cue and one in which the information was transferred after the cue. The information transferred before the cue would be relevant only on the lucky trials on which the transferred information was tested. Nevertheless, this would provide a second source of information that would contribute to performance on some trials. If the two sources of information were indeed available, then one should be able to distinguish the two sources by instructing an observer where to begin his or her transfer. To distinguish these sources was the goal of the following experiment.

The experiment was based on the line-length discrimination paradigm (Palmer, 1988). The task was to discriminate the length of one of the lines in the study stimulus. Observers judged whether the single line in the test stimulus was longer or shorter than the corresponding line 


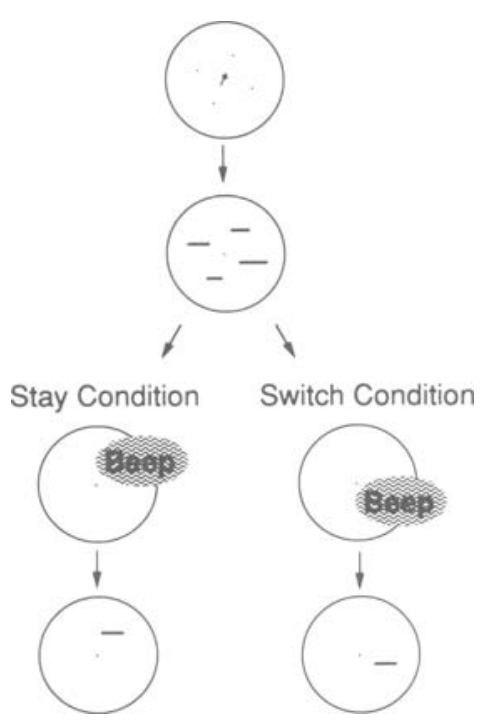

Figure 1. A schematic illustration of the experiment. An initial fixation display indicates which of the following stimuli are to be initially attended. This is followed by either the stay condition, in which the same location is cued and tested, or the switch condition, in which a different location is cued and tested.

in the study stimulus. The role of memory was manipulated through presentation of an auditory cue at some time before or after the study stimulus. For example, a lowfrequency, right-ear tone indicated the line in the lower right. This cue was always valid, and it allowed the observer to remember just a single line instead of all four. Observers were to use this cue to direct attention, but they were not to change their eye position. Eye fixation on the center of the display was to be maintained at all times.

The new feature of the experiment is illustrated in Figure 1. An initial fixation display contained an arrow that indicated to the observer that one particular location should be attended. As before, this was followed by the study stimulus and the auditory cue. The critical manipulation was whether or not the auditory cue marked the same position as that of the initial arrow instruction. In the stay condition, the auditory cue indicated the same position as that of the initial arrow; in the switch condition, the auditory cue indicated a different position. The probability of either condition was equal, so $50 \%$ of the time the initial attention instruction was appropriate. The auditory cue remained a $100 \%$ valid indicator of the final test position. The figure illustrates the condition in which the auditory cue followed the study display; in other conditions, the auditory cue occurred between the initial fixation display and the study display.

According to the transfer hypothesis, the initial position will always be transferred from the high-capacity store, so the stay condition will result in relatively good performance and will not be affected by cue delay. In the switch condition, performance will always be based on the transfer of information after the cue. Performance will decline with cue delay but will be as good as performance in the stay condition, if the cue is presented early enough.

\section{METHOD}

\section{Observers}

The 4 observers were adults from 20 to 40 years of age; all had normal or corrected-to-normal vision; and all were paid $\$ 5$ per hour, with the exception of Observer 1, the author. Data from the same observers, with the same identifying numbers, can be found in several related studies (Palmer, 1988, 1990; Palmer \& Ames, 1991).

\section{Apparatus}

A computer-controlled display was viewed from a distance of $78 \mathrm{~cm}$ through a $13-\mathrm{cm}$ aperture-reduction screen that was $20 \mathrm{~cm}$ in front of the display. Through the reduction screen, the visible area of the display was $12^{\circ}$ in diameter. All displays were black on white, using reverse video, which eliminates any phosphor persistence following stimulus offsets. The stimulus lines had a luminance of $70 \mathrm{~cd} / \mathrm{m}^{2}$, and the surround had a luminance of $170 \mathrm{~cd} / \mathrm{m}^{2}$, which yielded a contrast of 1.0:2.5. Further details can be found in Palmer (1988).

\section{Stimuli}

The stimulus sequence for a switch condition trial is illustrated in Figure 1. There were five successive displays: a fixation display, a blank display, a study display, a blank display (not shown), and a test display. The fixation display contained an arrow indicating which stimulus position was to be initially attended and was presented for $1,000 \mathrm{msec}$. The following blank interval was presented for $1,000 \mathrm{msec}$. The study stimulus contained the four lines and was presented for $50 \mathrm{msec}$. This was followed by the second blank display, which lasted $2,000 \mathrm{msec}$. Last of all was the test stimulus containing a single line, which was presented for $50 \mathrm{msec}$.

Auditory cues were presented once just before or just after the study display. Timing relative to the study stimulus is specified by cue delay, which is the interval between study stimulus offset and auditory cue onset. Cues after the stimulus offset have positive cue-delay values and are called postcues; cues before have negative values and are called precues. For example, a cue delay of $10 \mathrm{msec}$ means that the offset of the stimulus was followed $10 \mathrm{msec}$ later by the onset of the postcue tone. A cue delay of -100 msec means that the 50 -msec precue tone was followed immediately by the $50-\mathrm{msec}$ stimulus. Cues were presented to the right or left ear at 500 or $3000 \mathrm{~Hz}$. The ear of the tone's presentation indicated horizontal position, and the frequency of the tone indicated vertical position. For example, the upper left position was cued by highfrequency tone presented to the left ear.

The critical stimuli were horizontal lines that varied in length around a standard length of $60^{\prime}$ of arc. The lines were arranged on an imaginary circle $3^{\circ}$ from a central fixation point. The centers of the lines were placed on the circle at orientations of $1,4,7$, and 10 o'clock. In the test stimulus, the single line was always a standard of $60^{\prime}$ of arc. In the study stimulus, three lines were distractors, and one line was the comparison line. The length of the comparison line was selected from the values of $52^{\prime}, 54^{\prime}, 56^{\prime}, 64^{\prime}, 66^{\prime}$, and $68^{\prime}$ of arc, and the length of each distractor line was chosen randomly from the interval $50^{\prime}-70^{\prime}$ of arc.

\section{Procedure}

Observers were instructed to make a two-alternative forced choice discrimination of line length. Following the test display, observers pressed one key if the test line appeared shorter than the corresponding study line and another key if the test line appeared longer. There was no time pressure, and response accuracy feedback was provided by tones. The results were described as a psychometric function in which the proportion of "longer" responses is a function of the change in length. These data were fit to a cumulative normal, and the results were summarized by a threshold measure derived from the fitted function. Specifically, the difference threshold was defined as half of the difference between the length that produced the "longer" response $25 \%$ of the time and the length that produced the "longer" response $75 \%$ of the time. 
Cue delays of $-500,-200,10$, and 500 msec and the stay versus switch conditions were factorially combined and presented in a mixed list design. Observers had previously participated in the visual memory experiments described in Palmer (1988) and hence had several thousand trials of practice. In addition, they had at least one session of practice in this specific experiment. The 4 observers participated in 10 sessions with 8 conditions, yielding 320 trials per condition per observer.

\section{RESULTS}

Mean length difference thresholds are shown in Figure 2 as a function of cue delay. The curve parameter denotes the stay versus switch condition. For the stay condition, cue delay had little effect except at $-500 \mathrm{msec}$. For the switch condition, cue delay had a general effect across the range of delays. Thus, for cue delays of $-200 \mathrm{msec}$ and greater, the predictions of the transfer hypothesis were met: There was an effect of cue delay for the switch condition, but little effect of cue delay for the stay condition.

Two aspects of the data require more detailed analysis: the effect of cue delay for the -500 -msec condition, and the highly variable standard errors.

One possible reason for the improved performance in the -500 -msec cue delay condition is that observers were moving their eyes despite instructions to maintain fixation. To check this, two 32-trial sessions of the experiment were repeated with a judge watching each observer for eye movements (as previously reported in Palmer, 1988). In one session, observers were told to make intentional eye movements to the cued stimulus. These

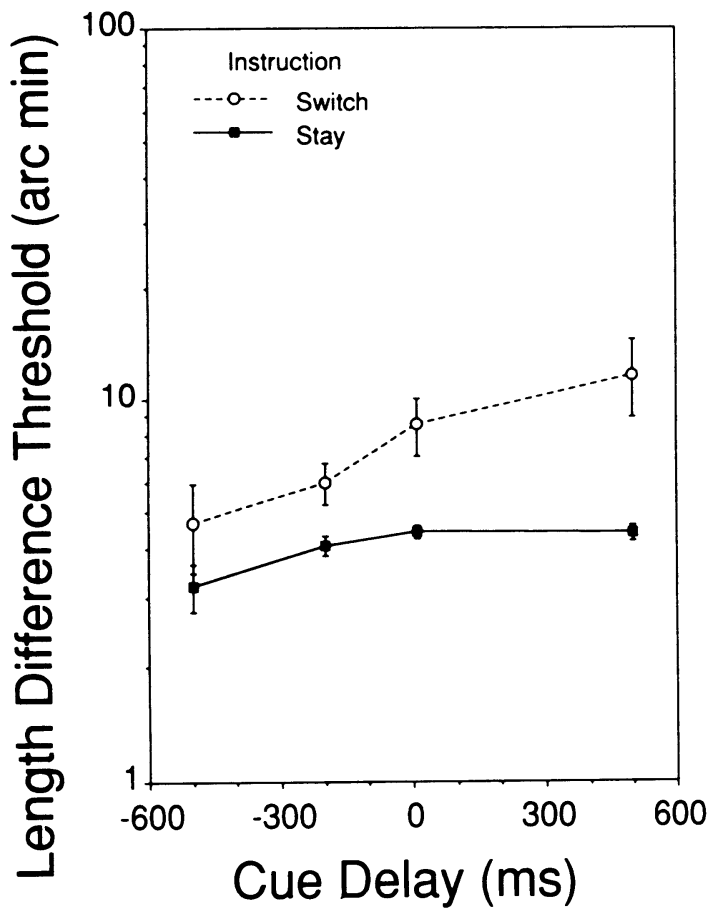

Figure 2. Mean length difference thresholds are shown as a function of cue delay for the two attention conditions. Error bars = $\pm S E M$.
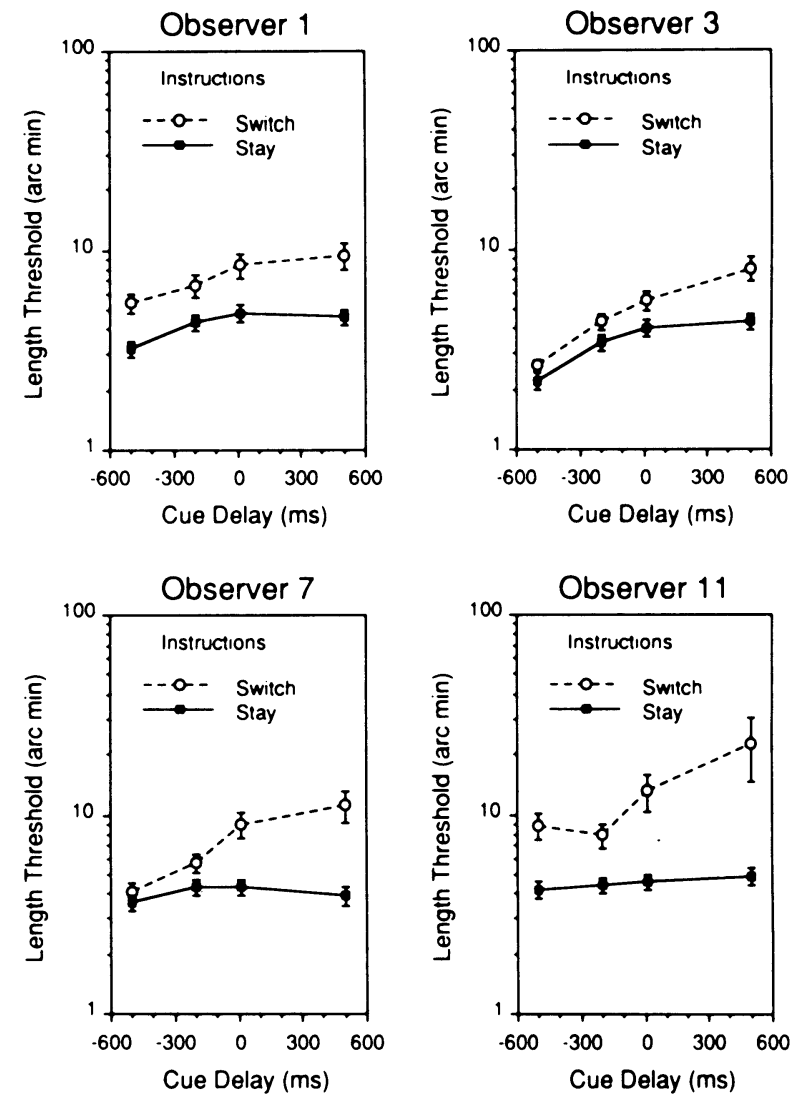

Figure 3. Length difference thresholds are shown as a function of cue delay and initial attention instruction. Each graph is for a separate observer.

roughly $3^{\circ}$ eye movements were detected on $94 \%$ of the trials. In the other session, observers were told to maintain fixation as the normal experiment. Observers 1,3 , 7 , and 11 were seen to make eye movements on $61 \%$, $89 \%, 0 \%$, and $25 \%$ of the trials. Only Observer 7 was successful in maintaining eye fixation. Other observers, including the author, made eye movements on some percentage of the trials. The demands of attending to one place and fixating another are no doubt at the root of the difficulty in maintaining fixation. This artifact makes it difficult to interpret results for the -500 -msec cue delay. Fortunately, this artifact does not affect the interpretation of the other cue delays, because for those cue delays there was not enough time to complete an eye movement before the end of the display.

The highly variable standard errors in Figure 2 are due to individual differences' being much larger in some conditions than in others. Graphs for individual observers are shown in Figure 3. Each graph shows the lengthdifference threshold as a function of cue delay for the stay and switch conditions. Consider the data of Observer 7, who was the best at following the eye-fixation instructions. His data follow the predictions most closely. There was an effect of cue delay for the switch condition, and there was little or no effect of cue delay for the stay condition. Furthermore, the stay and switch conditions have about 
the same threshold at a cue delay of $-500 \mathrm{msec}$. The other observers' data deviate from the predicted results in two ways. In the stay condition, Observers 1 and 3 show improvements with the -500 -msec cue delay that were probably due to their eye movements. For the other cue delays, their data follow the predictions. Observer 11 shows an advantage of the stay condition relative to the switch condition, which persists even with -500 -msec precues. This observer may have failed to use the auditory cue on some fraction of the trials or may have needed more time to interpret the sequence of attention cues. Together, the 4 observers show the closest agreement in the stay condition with the positive cue delays. This is why the standard errors in those conditions were so small in Figure 2. In the other conditions, the differences between observers lead to large standard errors of the mean threshold.

\section{DISCUSSION}

The experiment demonstrated that two components can be distinguished in a visual memory experiment. This was particularly clear in Observer 7, who was measured to be the best at following fixation instructions in this paradigm. For this observer, cue delay had an effect for the switch condition but little or no effect for the stay condition. Furthermore, the difference between stay and switch was eliminated when the cue preceded stimulus offset by $500 \mathrm{msec}$. Thus, this observer matched completely the predictions of the transfer hypothesis.

The specific predictions of the transfer hypothesis were not completely met for the other observers. Two observers showed cue delay effects with -500 -msec precues in both the stay and the switch conditions. This was almost certainly due to eye movements. For later cue delays, these observers showed the expected pattern of a cue-delay effect for the switch condition and no such effect for the stay condition. Another observer showed an overall advantage of the stay condition relative to the switch condition. This effect was probably due to occasional failures to use the auditory cue. Otherwise, this observer also showed the expected pattern of cue-delay effects in the switch condition and no such effect in the stay condition. More precise controls on observer strategies will be necessary to produce more uniform results. However, if one restricts attention to conditions in which observers had no chance to make eye movements, the predictions of the transfer hypothesis were met for all of the observers.

Further analysis of Observer 7 is possible by examining his results in a simple partial sampling experiment reported previously (Palmer, 1988), in which there was no effort to separate the components of visual memory. All three cue-delay effects follow the pattern expected from the transfer hypothesis: little or no cue-delay effect for the stay condition; the largest cue-delay effect for the switch condition; and an intermediate cue-delay effect for the no-instruction condition found in Palmer (1988). Such a noinstruction condition is probably measuring a mixture of the two components measured independently in the other conditions. Any detailed analysis of cue-delay effects needs to take into account the existence of these two components.

\section{Implications for Very-Short-Term Visual Memory}

The stay versus switch instruction manipulation changed the cue-delay effect despite the use of identical study and test stimuli. This implies that the cue-delay effect must be mediated by an attention-driven process and not by a stimulus-driven process. Being able to eliminate cue-delay effects by instruction rules out any possibility that the effect is due to stimulusdriven processes such as masking, changes in temporal uncertainty, or capacity demands of processing the cue. In summary, the mediation of the cue-delay effect by an attention variable eliminates any purely sensory hypotheses.

A second implication is relevant to interpreting the results within the context of the transfer hypothesis and its multiple memory stores. According to this hypothesis, the switch condition depends on only information transferred after the cue is presented. This condition satisfies the original goal of partial report experiments in measuring the amount of information available in the high-capacity store at the time of the cue. The difference between this condition and a no-instruction condition also suggests that previous measures did not distinguish these two components. Consequently, the current paradigm provides the best isolation yet of the highcapacity store.

\section{Alternatives to the Transfer Hypothesis}

Although the present results accord with the transfer hypothesis, they also accord with other hypotheses. In particular, the results also accord with the more general attentional hypothesis that stimuli are processed in two ways, which might be called attended and nonattended processing. Attended processing results in higher performance than does unattended processing, but this advantage declines with delay if attended processing is not initiated immediately. According to the transfer hypothesis, this temporal phenomenon is due to losses in the high-capacity store. More generally, other temporal processes may mediate this change in attention. For example, competing perceptual processes at each location may cumulatively interfere with one another (Di Lollo, 1980; Schulz, 1980), or attended processing may have limited capacity to establish associations within a single postcategorical store (Coltheart, 1980; van der Heijden, 1984). For these alternative hypotheses, the cue-delay effect is due to temporal properties of capacity limits other than limited transfer from a rapidly decaying high-capacity store. The lack of cue-delay effects in the stay condition indicates that instruction to attend a single item can overcome the capacity limitations, and the larger cue-delay effects in the switch condition are interpreted as measuring the remaining capacity at various delays. The new paradigm separates the two components of these alternative hypotheses, just as it separates the components of the transfer hypothesis.

\section{REFERENCES}

Coltheart, M. (1980). Iconic memory and visible persistence. Perception \& Psychophysics, 27, 183-228.

Di LoLLo, V. (1980). Temporal integration in visual memory. Journal of Experimental Psychology: General, 109, 75-97.

KINCHLA, R. A. (1973). Selective processes in sensory memory: A probecomparison procedure. In S. Kornblum (Ed.), Attention \& performance $I V$ (pp. 87-99). New York: Academic Press.

Palmer, J. (1988). Very short-term visual memory for size and shape. Perception \& Psychophysics, 43, 278-286.

PALmer, J. (1990). Attentional limits on the perception and memory of visual information. Joumal of Experimental Psychology: Human Perception \& Performance, 16, 332-350.

PALMER, J., \& AMES, C. T. (1991). Measuring the effect of multiple eye fixations on memory for visual attributes. Manuscript submitted for publication.

RUMELHART, D. E. (1970). A multicomponent theory of the perception of briefly exposed visual displays. Journal of Mathematical Psychology, 7, 191-218.

SCHULZ, T. (1980). Iconic memory-an artifact of perceptual reconstruction processes? Psychological Research, 42, 275-294.

SPERLING, G. (1960). The information available in brief visual presentations. Psychological Monographs: General \& Applied, 74, 1-29.

VAN DER Heuden, A. H. C. (1984). Postcategorical filtering in a barprobe task. Memory \& Cognition, 12, 446-457.

(Manuscript received March 7, 1991.) 